\title{
Raiva, Stress Emocional e Hipertensão: Um Estudo Comparativo
}

\author{
Glória de Fátima Araujo Moxotó ${ }^{1}$ \\ Marinha do Brasil \\ Lucia Emmanoel Novaes Malagris \\ Universidade Federal do Rio de Janeiro
}

\begin{abstract}
RESUMO - O estudo teve como objetivo investigar uma possível associação entre direção de expressão de raiva e stress em pessoas com hipertensão a partir da comparação com pessoas sem esse diagnóstico. Foram avaliados 112 participantes: 56 com hipertensão e 56 normotensos, pareados por escolaridade, gênero e faixa etária. Os instrumentos utilizados foram o Inventário de Sintomas de Stress para Adultos de Lipp e o Inventário de Expressão de Raiva como Estado e Traço. Participantes com hipertensão apresentaram 9,7 vezes mais chances de estarem estressados que normotensos $(O R=9,7 ; I C 95 \%: 4,0-23,5)$ e 19 vezes mais chances de expressarem raiva para dentro (OR=19; IC95\%:5,3-68).
\end{abstract}

Palavras-chave: raiva, stress, hipertensão

\section{Anger, Stress and Hypertension: A Comparative Study}

\begin{abstract}
This study aimed to investigate the association between the direction of the expression of anger and stress in persons with hypertension based on the comparison with persons without this diagnosis. One hundred and twelve (112) subjects were evaluated, fifty six (56) with hypertension and fifty six (56) without, matched for educational level, gender and age. The instruments were Lipp's Inventory of Stress Symptoms for Adults and the Inventory of Anger Expression as State and Trait. Hypertensive participants were 9.7 times more likely to be stressed than normotensive $(O R=9.7 ; 95 \%$ CI: 4.0-23.5) and 19 times more likely to express anger inward $(O R=19 ; 95 \%$ CI: 5.3-68).
\end{abstract}

Keywords: anger, stress, hypertension.

Ao lado de outros fatores como hereditariedade, vida sedentária e nutrição inadequada, stress e raiva estão relacionados à etiologia de diversas patologias crônicas e degenerativas como câncer, obesidade e hipertensão arterial (Straub, 2005). O stress pode ser compreendido como uma resposta do organismo da qual fazem parte fatores físicos, psicológicos, mentais e hormonais originada pela necessidade de manejo de qualquer situação, positiva ou negativa, que naquele momento representa ameaça (Lipp \& Malagris, 2011). Já a raiva, pode ser definida de acordo com Spielberger (1992) como um estado emocional que pode variar de mero aborrecimento ou irritação, até a fúria, sendo acompanhada por uma excitação do sistema nervoso autônomo.

Dentre as doenças citadas por sua associação com stress e raiva, a hipertensão arterial (HA), condição clínica multifatorial que se caracteriza por níveis elevados e sustentados de pressão arterial, apresenta grande expressividade epidemiológica em termos de saúde pública no Brasil. Esse quadro atinge em torno de $30 \%$ da população (SBC, SBH, \& SBN, 2010). Diante da magnitude desse problema, torna-se necessário conhecer melhor as relações entre os fatores envolvidos na origem, manutenção e agravamento da hipertensão arterial.

Uma das formas de compreender os fatores emocionais associados à hipertensão arterial, como é o caso do stress e da raiva, é o entendimento do conceito de reatividade cardiovascular, que se refere às modificações de pressão

1 Endereço para correspondência: Rua Figueiredo Magalhães 219 sala 1007, Copacabana, Rio de Janeiro, RJ, Brasil. CEP: 22.031-010.E-mail: gloriamoxoto@gmail.com arterial ou de frequência cardíaca, decorrentes de estímulos específicos. Embora a tendência geral da maioria das pessoas seja de demonstrar reatividade cardiovascular na forma de elevações de pressão arterial frente a situações de stress, indivíduos com hipertensão apresentam elevações maiores e mais frequentes do que pessoas sem este diagnóstico em situações similares. Por serem transitórias, essas elevações não produzem efeitos prejudiciais em indivíduos sem tendência à hipertensão, pois a capacidade normal de adaptação das artérias permite a recuperação do organismo sem ocasionar sequelas (Lipp \& Rocha, 2008).

Pode-se compreender o aumento de pressão sanguínea decorrente do stress como resultado da mediação de mudanças autonômicas e neuroendócrinas na contratibilidade cardíaca e na resistência vascular periférica. Essas alterações fisiológicas induzidas pelo stress fornecem suporte metabólico para o comportamento necessário à reação de luta e fuga. Alguns indivíduos apresentam, contudo, a tendência de demonstrar excessivos aumentos de pressão sanguínea, excedentes às demandas provenientes dos estressores.

Essas elevações excessivas de pressão sanguínea são preditoras de risco aumentado para hipertensão (Gianaros, Jennings, Sheu, Derbyshire, \& Matthews, 2007). Adicionalmente, Lipp e Rocha (2008) esclarecem que, nas pessoas que apresentam predisposição ao desenvolvimento da hipertensão, a frequente ativação do mecanismo orgânico é vista por muitos autores como responsável por produzir, pela repetição, um espessamento das paredes arteriais, o que ao longo do tempo, poderia atuar no desenvolvimento da hipertensão permanente. Assim, a reatividade cardiovascular excessiva tem sido apontada por vários autores (Pointer 
et al., 2012; Treiber et al., 2003), como capaz de atuar na patofisiologia da hipertensão arterial.

No que se refere ao stress, pesquisas têm investigado suas repercussões nas duas condições. Diversos autores investigaram a associação entre stress e reatividade cardiovascular (Gianaros et al. 2007; Lipp, Pereira, Justo, \& Matos, 2006; Nealey-Moore, Smith, Uchino, Hawkins, \& Olson-Cerny, 2007). Associações entre stress emocional e hipertensão foram também investigadas por diferentes autores, em pesquisas com enfoques variados (Gerin et al., 2012; Linden, Lenz \& Con, 2001). Complementarmente, as VI Diretrizes Brasileiras de Hipertensão Arterial apontam a necessidade do treinamento do controle do stress para pessoas com hipertensão (SBC, SBH, \& SBN, 2010).

Ao abrir uma nova dimensão para o estudo da associação entre stress e hipertensão, Malagris et al. (2009) verificou mudanças de transporte celular em mulheres com hipertensão após Treino de Controle do Stress (TCS). O referido autor observou redução do stress nas participantes com hipertensão, restaurando o transporte de L-arginina através do sistema $\mathrm{y}+$ em níveis apresentados em pacientes com hipertensão não-estressadas. Convém ressaltar que a L-arginina é um aminoácido precursor da produção de óxido nítrico (NO), gás vasodilatador (Malagris et al., 2009).

De forma similar, a raiva tem sido correlacionada à hipertensão (Player, King, Mainous, \& Geesey, 2007; Spielberger, 1992). Muitos desses estudos investigaram especificamente, a associação da direção da expressão de raiva com a ocorrência de hipertensão e com a reatividade cardiovascular. Segundo Spielberger (1992), a maneira pela qual a raiva é expressa, é uma variável crítica para o entendimento do papel dessa emoção, em sua associação frequente com patologias como a hipertensão. Assim, têm sido estudados na literatura dois estilos de expressão de raiva: para fora (RPF), ou seja, direcionada a outras pessoas e objetos do meio; e para dentro (RPD), em forma de supressão, ruminação, ressentimentos e mágoas. Indivíduos que apresentam simultaneamente os dois estilos, RPF e RPD, são classificados como RPDF (raiva para dentro e para fora).

Alguns autores defendem a relação do aumento da reatividade cardiovascular com a expressão de raiva para fora (Sanz et al., 2010), outros concluem que tal aumento está relacionado com a expressão da raiva para dentro (Poole, Snieder, Davis, \& Treiber, 2006; Richter et al., 2011). Já Lipp (2005), a partir de pesquisas, concluiu que, independente do estilo de expressão da raiva ser para fora ou para dentro, quando é muito frequente, se mostra associado a aumentos da reatividade cardiovascular. Em relação à hipertensão, a tendência a suprimir constantemente a raiva, direcionando-a para dentro, vem sendo associada positivamente a essa doença (Hosseini, Mokhberi, Mohammadpour, Mehrabianfard, \& Lashak, 2011; Ohira, 2010).

Além de considerar a raiva e suas formas de expressão na relação com a reatividade cardiovascular e a hipertensão, é necessário enfatizar que essa emoção, quando expressa frequentemente para fora ou para dentro, também pode atuar como um fator corrosivo nos relacionamentos interpessoais, contribuindo para o prejuízo da qualidade de vida do indivíduo (Lipp, 2005). A raiva excessiva, uma vez que prejudica as relações sociais, pode interferir negativamente na manutenção de apoio social, que é um fator protetor contra o adoecimento e auxilia na reabilitação de grande parte das patologias (Gallant, 2003). Corroborando estas proposições, Parrot, Zechner e Evces (2005) afirmam que, mesmo na ausência de um diagnóstico clínico, a raiva pode interferir de forma negativa no funcionamento social.

Adicionalmente, uma meta-análise realizada por Chida e Hamer (2008) incluiu 729 estudos realizados num período de 30 anos, que investigavam entre amostras de populações saudáveis, a associação entre fatores psicossociais e resposta a stress induzido em laboratório. Os autores identificaram uma associação positiva entre raiva, hostilidade, e elevações da reatividade cardiovascular.

A partir dos resultados dos estudos citados, podem ser constatadas interseções entre stress, raiva e hipertensão que demandam análise simultânea visando esclarecer lacunas ainda existentes sobre as relações entre essas três variáveis que parecem potencializar-se mutualmente. Desta forma, o presente estudo teve como objetivo investigar uma possível associação entre direção de expressão de raiva e stress em pessoas com hipertensão a partir da comparação com pessoas sem este diagnóstico.

\section{Método}

\section{Participantes}

Participaram do estudo 112 indivíduos voluntários, sendo 56 com hipertensão, nos estágios 1 (hipertensão leve) e 2 (hipertensão moderada), atendidos no Programa de Hipertensão e Diabetes de um centro de saúde municipal do Rio de Janeiro. O estudo teve aprovação do Comitê de Ética e Pesquisa da Secretaria Municipal de Saúde e Defesa Civil. Participaram, ainda, 56 indivíduos sem diagnóstico de hipertensão, acompanhantes de pacientes do referido centro de saúde.

Os critérios de inclusão para a amostra foram: ter entre 30 e 65 anos, possuir no mínimo nível fundamental incompleto e no máximo nível médio de escolaridade, não estar fazendo uso de psicofármacos, não ser portador de diabetes, não ter sofrido infarto do miocárdio ou acidente vascular cerebral (AVC), não sofrer de doença renal crônica, não ser portador de cardiopatia isquêmica. Os participantes com hipertensão estavam em tratamento médico. Os dois grupos (com e sem hipertensão) foram pareados em variáveis como: faixa etária, sexo e escolaridade.

\section{Instrumentos}

Foram utilizados o Inventário de Sintomas de Stress para Adultos de Lipp (ISSL) (Lipp, 2000) e o Inventário de Expressão de Raiva como Estado e Traço (STAXI) (Spielberger, 1992). O ISSL baseia-se no modelo quadrifásico do stress, fornecendo o diagnóstico em fases (alerta, resistência, quase-exaustão e exaustão). O ISSL é composto por três quadros que contêm sintomas físicos e psicológicos de cada fase do stress. A Tabela 1 apresenta os sintomas da 
$1^{\mathrm{a}}$ fase do stress, a Tabela 2 , os sintomas das $2^{\mathrm{a}}$ e $3^{\mathrm{a}}$ fase e a Tabela 3, da quarta fase. O ISSL contém 34 itens de sintomas físicos e 19 de sintomas psicológicos.

Foram realizadas análises estatísticas de validação do ISSL a partir das respostas de 1843 participantes voluntários da comunidade, divididos entre mulheres (64\%) e homens (36\%). A análise de confiabilidade foi aplicada tendo como resultado o coeficiente de Alfa de 0,9121, o que corresponde à expressiva confiabilidade do inventário, ou seja, os itens correspondem ao verdadeiro valor para o conceito intencionado, ou seja, mensurar o nível de stress. Foi também utilizada a extração fatorial pelo método de correspondências múltiplas, demonstrando-se dois eixos fatoriais de importância na determinação das relações dos itens, com grandes contribuições nos fatores, que foram designados sintomas físicos e psicológicos (Lipp, 2000).

O STAXI é formado por 44 itens que compõem seis escalas (Estado, Traço, Raiva para Fora, Raiva para Dentro, Controle e Expressão) e duas sub-escalas da escala de traço (temperamento e reação de raiva). A consistência interna das escalas foi avaliada mediante o alfa de Cronbach tendo sido encontrados valores altos entre 0,69 a 0,88 (Spielberger, 1992). Dentre os componentes da raiva avaliados pelo STAXI, foram utilizados neste estudo, para atingir os objetivos propostos, os resultados relativos a: RPD e a RPF acima da média, e RPDF, nos casos onde houve ocorrência simultânea num mesmo indivíduo, de RPD e RPF acima da média. Os valores médios constam no manual do instrumento.

\section{Procedimentos}

Após a análise dos dados contidos nos prontuários de pacientes com hipertensão que tinham consulta marcada no mesmo dia, foi feita a seleção daqueles que atendiam aos critérios de inclusão da pesquisa. Os participantes foram informados sobre os procedimentos envolvidos na pesquisa e aqueles que se dispuseram a participar em caráter voluntário, com e sem hipertensão, assinaram o Termo de Consentimento Livre e Esclarecido (TCLE) e foram então submetidos à aplicação do ISSL e do STAXI.

Os dados sociodemográficos (sexo, idade, escolaridade e estado civil) de todos os participantes foram obtidos com as informações fornecidas pelos dados de identificação constantes nas folhas de rosto do ISSL e STAXI. Após a aplicação dos inventários, estes foram corrigidos e os resultados transmitidos individualmente aos participantes em entrevistas de devolução agendadas duas semanas depois da coleta de dados. Tanto a aplicação dos instrumentos quanto a transmissão dos resultados foram feitas individualmente. Todos os participantes obtiveram, como benefício, o recebimento de orientações sobre o manejo do stress e da raiva.

\section{Análise de dados}

Foi usado o software SPSS 17 para a análise de dados. Foram utilizados para detecção da existência de diferenças significativas das proporções/médias, entre os grupos de participantes (com e sem hipertensão), em função das exposições de interesse: stress e direção da expressão de raiva (RPD, RPF, RPDF) testes qui-quadrado, quando comparadas as variáveis categóricas duas a duas, e ANOVA e teste pós Hoc de Bonferroni para as variáveis contínuas quando comparadas as médias entre os grupos. O nível de significância adotado para todos os testes estatísticos foi de $5 \%$, ou seja, $p<0,05$. Com o objetivo de identificar especificamente para as variáveis binárias se algum dos grupos apresentava mais chances de ter/não ter stress, ou ter/não ter RPD, RPF, RPDF acima da média, calculou-se também a razão dos produtos cruzados ( "Odds Ratio"- OR) e seus respectivos intervalos de confiança (IC 95\%), ao nível de significância de $5 \%$.

\section{Resultados}

Os dados sociodemográficos revelaram que, quanto ao sexo, houve uma expressiva representatividade de participantes do sexo feminino $(78,6 \%)$, em relação aos participantes do sexo masculino $(21,4 \%)$. Já em relação à faixa etária, os participantes cuja idade variava entre 51 a 65 anos $(50,0 \%)$ anos tiveram a maior frequência da amostra, seguidos pelos de 41 a 50 anos $(37,5 \%)$ e os de 30 a 40 anos (12,5\%). A maioria dos participantes possuía nível de escolaridade fundamental completo $(76,8 \%)$, seguidos pelos que possuíam médio incompleto $(14,3 \%)$ e fundamental incompleto $(8,9 \%)$. Quanto ao estado civil, houve maior frequência de casados $(73,2 \%)$, enquanto o restante da amostra se distribuiu entre separados/divorciados $(11,6 \%)$, solteiros $(8,9 \%)$ e viúvos $(6,3 \%)$.

Quanto ao stress, de acordo com os resultados do ISSL, verificou-se que $64(57,1 \%)$ dos 112 participantes estavam estressados, sendo que nenhum deles obteve escores referentes às fases de alerta ou exaustão. No que se refere à pressão arterial (com e sem hipertensão), dentre os 56 com hipertensão, $46(82,1 \%)$ apresentavam stress, já dentre os 56 sem HA, $18(32,1 \%)$ estavam estressados. A análise estatística revelou diferença significativa $(p=0,000)$ entre os participantes com e sem hipertensão desta amostra no que se refere ao stress. Os participantes com hipertensão apresentaram 9,7 vezes mais chances de estarem estressados que aqueles sem este diagnóstico $(O R=9,7 ; I C 95 \%: 4,0-23,5)$

O STAXI possibilitou identificar a direção da expressão de raiva (para dentro- RPD; para fora-RPF; para dentro e para fora-RPDF) na amostra total, e separadamente, em participantes com e sem hipertensão, estressados e não estressados. Considerando a amostra total $(\mathrm{N}=112)$, verificou-se que $59(52,7 \%)$ dentre os 112 participantes apresentaram RPD $(N=32), \operatorname{RPF}(N=12)$ e RPDF $(N=15)$ acima da média. Todos esses 59 participantes estavam estressados. No entanto, apenas $5(7,8 \%)$ dentre os 64 participantes estressados não apresentaram direção de expressão de raiva acima da média. Ficou evidente, então, que $92,2 \%$ (59) dos 64 participantes estressados da amostra, apresentaram índices de RPD, RPF e RPDF acima da média.

Foi feita comparação entre os participantes com e sem hipertensão relativa à direção de expressão de raiva. Verificou-se que houve diferença significativa $(p=0,000)$ 
referente ao índice de expressão de RPD, pois enquanto 29 participantes com hipertensão $(51,8 \%)$ apresentaram índices de RPD acima da média, apenas três participantes sem hipertensão $(5,4 \%)$ apresentaram índices de RPD acima da média $(p=0,000)$. Desta forma, os participantes com hipertensão apresentaram 19 vezes mais chances de ter RPD acima da média do que os sem hipertensão $(O R=19$; IC95\%:5,3-68).

Houve uma associação inversa com a hipertensão quanto à expressão de RPF, com 11 (19,6\%) participantes sem hipertensão apresentando resultados acima da média, contra somente um participante com hipertensão $(1,8 \%)$, configurando diferença estatisticamente significativa $(p=$ 0,002). Assim, participantes sem hipertensão apresentaram 13,4 vezes mais chances de terem RPF acima da média do que os com hipertensão $(O R=13,4 ; I C 95 \%: 1,67-108,12$.)

Os resultados da expressão de RPDF obtidos pela observação da elevação simultânea de RPD e RPF também foram significativamente diferentes $(p=0,000)$, com 12 $(21,4 \%)$ dos participantes com hipertensão acima da média, contra três $(5,4 \%)$ dos participantes sem hipertensão. Desta maneira, os participantes com hipertensão apresentaram 4,8 vezes mais chances que os demais de ter RPDF acima da média $(O R=4,8 ; I C 95 \%: 1,3-18,2)$.

Para a investigação da associação entre stress e direção de expressão de raiva em participantes com e sem hipertensão, foram comparadas as médias dos escores T dos participantes divididos em quatro grupos: sem hipertensão e sem stress $(n=38)$, sem hipertensão estressados $(n=18)$, com hipertensão sem stress $(n=10)$, com hipertensão e estressados $(n=46)$. O manual do STAXI fornece os escores T correspondentes aos escores brutos. Uma vez que os escores $\mathrm{T}$ se baseiam em transformações lineares normalizadas dos escores brutos de cada escala, eles se configuram como mais adequados para satisfazer suposições básicas da maior parte das análises estatísticas.

A média mais elevada de escores T em RPD foi identificada entre os com hipertensão e estressados (Tabela 1), constatando-se diferença significativa $(p=0,000)$ em relação aos sem hipertensão e estressados. O inverso foi observado em relação à RPF, cujos escores foram mais elevados entre os sem hipertensão e estressados, verificando-se diferença estatisticamente significativa na comparação com os participantes com hipertensão e estressados (Tabelas 1 e 2). Os resultados da direção de expressão de RPDF não foram incluídos por serem obtidos mediante índices simultâneos acima da média nas escalas de RPD e RPF. Desta forma, uma vez que não há uma escala de RPDF, os escores T não são disponibilizados. A Tabela 2 complementa as informações da Tabela 1 no sentido de apresentar quais médias são significativamente diferentes nos grupos dois a dois.

Tabela 1. Média dos ${ }^{a}$ escores-T e p-valor da ANOVA segundo RPD e RPF nos grupos de participantes com e sem hipertensão, com e sem stress

\begin{tabular}{lccccc}
\hline & \multicolumn{2}{c}{ Média - Valor (T) } & \multicolumn{2}{c}{ P-Value } \\
RH sem & CH sem & SH & CH & Anova \\
\hline RPD (Valor T) & stress $n=38$ & stress $n=10$ & estressado $n=18$ & estressado $n=46$ & 0,000 \\
RPF (Valor T) & 44,7 & 36,9 & 47,9 & 63,5 & 0,000 \\
Participantes (n) & 37,1 & 34,2 & 59,6 & 45,0 & \\
\hline
\end{tabular}

Nota. $\mathrm{SH}=$ sem hipertensão; $\mathrm{CH}=$ com hipertensão

${ }^{\text {a }}$ Fonte:Manual STAXI

Tabela 2. P-valores no teste pós Hoc de Bonferroni (significância), segundo RPD e RPF nos grupos de participantes, a partir da Tabela 1

\begin{tabular}{|c|c|c|c|c|}
\hline & \multicolumn{4}{|c|}{ p-valores no texte pós-Hoc de Bonferroni } \\
\hline & SH e sem stress & CH sem estress & SH estressado & CH estressado \\
\hline \multicolumn{5}{|l|}{ RPD } \\
\hline SH e sem stress & - & - & - & - \\
\hline $\mathrm{CH}$ sem estress & 0,100 & - & - & - \\
\hline SH estressado & 1,000 & $0,014^{*}$ & - & - \\
\hline $\mathrm{CH}$ estressado & $0,000 *$ & $0,000 *$ & $0,000 *$ & - \\
\hline \multicolumn{5}{|l|}{ RPF } \\
\hline SH e sem stress & - & & & \\
\hline $\mathrm{CH}$ sem estress & 1,000 & & & \\
\hline SH estressado & $0,000 *$ & $0,000^{*}$ & & \\
\hline $\mathrm{CH}$ estressado & $0,016^{*}$ & 0,06 & $0,000 *$ & \\
\hline
\end{tabular}

Nota. $\mathrm{SH}=$ sem hipertensão; $\mathrm{CH}=$ com hipertensão $*^{*} p<0,05$ 


\section{Discussão}

O estudo investigou a associação entre stress e hipertensão. $\mathrm{O}$ fato de participantes com hipertensão terem apresentado 9,7 vezes mais chances do que os demais de estarem estressados, confirma dados existentes na literatura sobre a associação positiva entre stress e hipertensão (Fiorotti, Tomazelli, \& Malagris, 2009; Gasperin, Netuveli, Diasda-Costa, \& Patussi, 2009; Lipp \& Malagris, 2001; Lipp \& Rocha, 2008; Malagris et al., 2009). Tais resultados estão de acordo com as VI Diretrizes Brasileiras de Hipertensão (SBC, SBH, \& SBN, 2010), que identificam o stress emocional como capaz de atuar na elevação transitória da reatividade cardiovascular e da pressão arterial.

Além da proposição de que o stress influi na origem, manutenção e agravamento da hipertensão, pode-se refletir sobre o fato da hipertensão já estabelecida se constituir em uma fonte de stress para o portador da mesma, processo que configura um círculo vicioso (Malagris, 2004). Se por um lado o stress excessivo pode atuar em aumentos constantes da reatividade cardiovascular (Gianaros et al., 2007; Gianaros $\&$ Sheu, 2009; Treiber et al., 2003), por outro lado pode-se inferir que as condições relacionadas à hipertensão tornem a rotina do portador dessa patologia significativamente mais estressante. Desta maneira, a convivência com o diagnóstico e suas consequências, tais como o controle da medicação, a incerteza diante do curso da doença e o risco de perda do controle da pressão aliados ao temor das possíveis complicações decorrentes e da morte, são passíveis de ocasionar um decréscimo na qualidade de vida da pessoa hipertensa (Malagris, 2004).

De forma semelhante ao stress, a ocorrência de RPD acima da média apresentou uma associação positiva com a hipertensão. Os participantes com este diagnóstico apresentaram 19 vezes mais chances de ter RPD acima da média do que os participantes sem hipertensão. Esses resultados corroboram pesquisas de outros autores (Spielberger, 1992; Treiber et al., 2003) que demonstraram a associação entre a expressão de RPD e hipertensão arterial, e a relacionaram a aumentos de reatividade cardiovascular. Os resultados de uma pesquisa realizada sobre os efeitos da ruminação de raiva, característica da expressão de RPD, na reatividade cardiovascular em indivíduos hipertensos, são concordantes aos obtidos no presente estudo. Os autores concluíram que, além da excitação momentânea induzida pela situação provocadora de raiva, a ruminação mantém posteriormente essa excitação e interfere na recuperação espontânea e, consequentemente, prolonga o aumento da reatividade cardiovascular (Gerin et al., 2012).

Os dados obtidos no presente trabalho sobre a predominância de RPD entre hipertensos podem, ainda, ser relacionadas tanto à falta de assertividade quanto à alexitimia. Embora tais características não tenham sido objeto do estudo, pesquisas anteriores demonstraram que essas condições são comumente presentes em muitos indivíduos com hipertensão e que influenciam a magnitude da reatividade cardiovascular (Lipp, Frare, \& Santos, 2007). Os resultados aqui encontrados podem, portanto, ter como influência a inassertividade e a alexitimia, cuja combinação, provavelmente, interfere na expressão da raiva, configurando o elevado índice de RPD.
Outro ponto importante merece ênfase no que se refere à RPD. Estamos nos referindo a fator que é uma característica de muitas pessoas com hipertensão de tentar manter controle sobre tudo o que se passa a sua volta, inclusive a expressão de suas emoções (Lipp \& Rocha, 1994). De fato, embora inibir emoções tenha efeito sobre a elevação da reatividade cardiovascular de hipertensos, já foi demonstrado que, comparativamente, expressar emoções de forma inadequada tem efeitos significativamente mais altos na elevação da reatividade cardiovascular (Lipp, Pereira, Justo, \& Matos, 2006). Assim, se inibir é prejudicial, externar pode ser mais deletério ainda.

É possível, então, que inibir emoções exerça um efeito protetor imediato em pessoas com hipertensão e, assim, a busca da expressão adaptativa para essas emoções, por meio de intervenções psicológicas, deve levar em conta essa característica. Existe, ainda, a possibilidade de que a direção da expressão de raiva seja também influenciada pela aprendizagem, decorrente da observação dos estilos parentais (Lipp, 2005). Uma vez que a hipertensão tem um forte componente hereditário, é possível que muitos indivíduos com hipertensão tenham se desenvolvido em lares onde a expressão de RPD é predominante.

Interessante também foi o resultado encontrado quanto à expressão de RPDF acima da média, obtida, como já foi mencionado, através da junção dos resultados das escalas de RPD e RPF simultaneamente acima da média. Participantes com hipertensão apresentaram 4,8 vezes mais chances de ter RPDF acima da média que os demais participantes. Segundo a literatura, essa forma de expressão da raiva está associada à elevação do risco de ataques cardíacos (Kassinove \& Tafrate, 2007; Spielberger, 1992). Logo, pode-se inferir que importante parcela da amostra estudada pode apresentar risco de ocorrência de ataques cardíacos, em parte associado ao estilo de expressão de raiva.

Embora a RPD seja a forma predominante de expressão entre indivíduos com hipertensão, o fato de que RPDF simultaneamente tenha se mostrado mais presente entre os que apresentavam este diagnóstico do que entre os que não o apresentavam, leva a reflexões. É possível que pessoas com hipertensão habituadas a guardar a raiva, apesar dos esforços constantes, em alguns momentos não consigam fazê-lo, devido ao acúmulo de tensão relacionado a essa contenção. Nesse caso, é possível que ocorram em situações alternadas às contenções, explosões que podem trazer sentimentos de culpa e stress, o que leva à não obtenção do alívio real com a expressão da emoção de raiva. Após as explosões, voltam então a conter a raiva até o próximo comportamento explosivo e assim por diante, sem chegar a um meio termo que seria o manejo adaptativo da raiva.

Já a expressão de RPF acima da média apresentou associação inversa com a hipertensão, confirmando dados da literatura (Spielberger, 1992). Os participantes sem hipertensão apresentaram 13,4 vezes mais chances de terem RPF acima da média que os com essa patologia. Tais resultados são concordantes com a literatura, ao indicarem que portadores de hipertensão tendem ou a somente inibir e ruminar sentimentos de raiva, ou a alternar a inibição com a expressão de RPF, ambas de forma excessiva (Spielberger, 1992). 
É possível que participantes sem hipertensão desta amostra tenham apresentado RPF acima da média mais frequentemente do que os participantes com hipertensão porque estes, na maioria das vezes, parecem expressar para fora apenas quando também expressam a RPD alternativamente. Tal fato parece ser decorrente de uma inibição exagerada da raiva no seu dia a dia, resultando em explosão e sentimentos de culpa, como já aqui discutido.

Verificou-se, nesta amostra, que os 59 participantes que apresentaram direção de expressão de raiva acima da média, seja RPD, RPF ou RPDF, quando comparados em relação à associação entre stress e raiva, estavam estressados. Nem todos os 64 estressados, porém, apresentaram RPD, RPF ou RPDF acima da média. Os resultados encontrados são coerentes com a afirmação de Lipp (2005), que sustenta que nem todas as pessoas que têm stress excessivo têm raiva. Pode-se compreender a afirmação da autora, no sentido de que estar estressado não equivale obrigatoriamente a estar vivenciando ou expressando excessivamente a raiva, pois nem todos os estressores estão ligados a essa emoção. Da mesma forma, nem todos os indivíduos estressados exibirão propensão à expressão excessiva dessa emoção. A situação estressora pode estar ligada a diversas emoções e sentimentos tais como medo, ansiedade, frustração, ciúmes e inveja, entre outros (Lipp, 2009).

Lipp (2005) argumenta, no entanto, que é provável que os indivíduos que apresentem índices de raiva acima da média, configurando uma expressão excessiva, apresentem stress simultaneamente. Essa proposição pode ser compreendida a partir do entendimento do processo de desenvolvimento da raiva, no qual, após a avaliação inicial do estímulo desencadeante, o stress é sempre deflagrado, preparando o indivíduo para enfrentar a situação inicial, seja expressando ou inibindo a emoção (Lipp, 2005). A seguir, o estímulo é reavaliado já sob a influência da raiva, ocorrendo então, o seu escalonamento, contexto em que o stress emocional, com suas manifestações físicas e psicológicas, e a raiva, vão potencializar-se mutuamente.

Cabe ressaltar que stress e raiva deflagram mecanismos análogos. Assim, a presença do stress e suas consequentes alterações fisiológicas e hormonais em indivíduos que já tenham a tendência de avaliar as situações como injustas ou desagradáveis, e que reagem a elas com raiva, provavelmente torna-os mais vulneráveis a vivenciar essa emoção. É importante registrar que uma vez associados, raiva e stress, podem provocar uma exacerbação recíproca, e contribuir para elevar a reatividade cardiovascular, bem como para deteriorar a qualidade de vida do hipertenso.

Verificou-se que a associação entre stress e RPD acima da média foi mais frequente entre participantes com hipertensão que nos demais, encontrando-se, a partir da análise estatística, diferença significativa entre os dois grupos $(p=0,000)$. O inverso foi observado em relação à associação entre stress e RPF, com maior frequência entre o grupo sem hipertensão, configurando também diferença estatisticamente significativa. Os efeitos da associação entre stress e RPD ocasionam impacto direto na rotina do indivíduo com hipertensão, uma vez que a inibição excessiva de raiva pode afetar as relações interpessoais, dificultando a clareza da comunicação pelo prejuízo do entendimento.
Ao suprimir frequentemente a raiva, o indivíduo com hipertensão, muitas vezes, deixa de fazer a justa reivindicação dos próprios direitos, defesa de suas opiniões e desejos. Em decorrência, as pessoas que o cercam, desconhecem suas reais opiniões e emoções. Além disso, a inibição da raiva é comumente acompanhada por uma expressão facial contrariada e insatisfeita. Considerando que o reconhecimento da expressão facial de raiva é ágil e imediato por seu valor adaptativo (Fox et al., 2000), a expressão de contrariedade pode ser interpretada por quem estiver envolvido na interação como uma ameaça, provocando afastamento ou gerando comportamento agressivo em revide. Portanto, como as reações das pessoas ao redor não foi avaliada, essa situação pode ser considerada como uma limitação deste estudo. Sugere-se que essa variável possa ser abordada em pesquisas futuras.

Em conclusão, cabe ressaltar que uma vez que uma comunicação clara é necessária para promover relações interpessoais gratificantes e funcionais, falhas na comunicação decorrentes da excessiva inibição de raiva podem ser consideradas preditoras de uma deterioração na qualidade de vida na área social da pessoa com hipertensão, incluindo tanto os contextos familiares e conjugais, como os ocupacionais. Esse prejuízo é passível de ocasionar stress, que por sua vez, poderá atuar exacerbando a raiva. A saúde dessa pessoa também será afetada, pois se ocorrerem de forma excessiva, tanto a inibição da raiva quanto o stress, podem ocasionar elevação da reatividade cardiovascular, e assim, aumentar o impacto negativo da associação entre o stress e a RPD, em todas as áreas da vida do indivíduo com hipertensão.

\section{Referências}

Chida, Y., \& Hamer, M. (2008). Chronic psychosocial factors and acute physiological responses to laboratory-induced stress in healthy populations: a quantitative review of 30 years of investigations. Psychological Bulletin, 134(6), 829-885.

Fiorotti, C., Tomazelli, J., \& Malagris, L. E. N. (2009). Transtornos mentais comuns em pacientes hipertensos: estudo em unidade de atenção primária á saúde no Rio de Janeiro. Revista APS, 12(3), 318-327.

Fox, E., Lester, V., Russo, R., Bowles, R. J., Pichler, A., \& Dutton, K. (2000). Facial expressions of emotion. Are angry faces detected more efficiently? Cognition \& Emotion, 14(1), 61-92.

Gallant, M. P. (2003). The influence of social support on chronic illness self-management: a review and directions for research. Health Education \& Behaviour, 30(2), 170-195.

Gasperin, D., Netuveli, G., Dias-da-Costa, J. S., \& Patussi, M. P. (2009). Effects of psychological stress on blood pressure increase: a meta analysis of cohort studies. Cadernos de Saúde Pública, 25(4), 715- 726.

Gerin, W., Zawadzki, M. J., Brosschot, J. F., Thayer, J. F., Christenfeld, N. J., Campbell, T. S., \& Smyth, J. M. (2012). Rumination as a mediator of chronic stress effects on hypertension: a causal model. International Journal of Hypertension, 1-9. doi:10.1155/2012/453465. 
Gianaros, P. J., Jennings, J. R., Sheu, L. K., Derbyshire, S. W., \& Matthews, K. A. (2007). Heightened functional neural activation to psychological stress covaries with exaggereted blood pressure reactivity. Hypertension, 49(1), 134-140.

Gianaros, P. J., \& Sheu, L. K. (2009). A review of neuroimaging studies of stressor evoked blood pressure reactivity: Emerging evidence for a brain body pathway to coronary disease risk. Neuroimage, 47(3), 922-936.

Hosseini, S. H., Mokhberi, V., Mohammadpour, R.A., Mehrabianfard, M., \& Lashak, N. B. (2011). Anger expression and suppression among patients with essential hypertension. International Journal of Psychiatry Clinical Practice, 15(3), 214-218.

Kassinove, H., \& Tafrate, R. C. (2007). Anger Management: The complete guidebook for practitioners. Atascadero, California: Impact Publishers.

Linden, W., Lenz, J.W., \& Con, A.H. (2001). Individualized stress management for primary hypertension: a randomized trial. Archives of Internal Medicine, 161(8),1071-80.

Lipp, M. E. N. (2000). Manual do Inventário de Sintomas de Stress para Adultos de Lipp (ISSL). São Paulo: Casa do Psicólogo.

Lipp, M. E. N. (2005). Stress e o turbilhão da raiva. São Paulo: Casa do Psicólogo.

Lipp, M. E. N. (2009) Sentimentos que causam stress: como lidar com eles. Campinas: Editora Papirus.

Lipp, M. E. N., Frare, A., \& Santos, F. U. (2007). Efeitos de variáveis psicológicas na reatividade cardiovascular em momentos de stress emocional. Estudos de Psicologia, 24(2), 161-167.

Lipp, M. E. N., Pereira, M. M. B., Justo, A. P., \& Matos, T. M. G. (2006). Cardiovascular reactivity in hypertensives: Differential effect of expressing and inhibiting emotions during moments of interpersonal stress. The Spanish Journal of Psychology, 9(2), 154-161.

Lipp, M. E. N., \& Malagris, L. E. N. (2001). O stress emocional e seu tratamento. In B. Rangé (Ed.), Psicoterapias CognitivoComportamentais: Um diálogo com a Psiquiatria (pp. 475490). Porto Alegre: Artmed.

Lipp, M. E. N., \& Malagris, L. E. N. (2011). Estresse: aspectos históricos, teóricos e clínicos. In B. Rangé (Ed.), Psicoterapias Cognitivo-Comportamentais: Um diálogo com a Psiquiatria (pp. 617-632). Porto Alegre: Artmed.

Lipp, M. E. N., \& Rocha, J. C. (1994). Stress, hipertensão arterial e qualidade de vida: um guia de tratamento para o hipertenso. Campinas: Papirus.

Lipp, M. E. N., \& Rocha, J. C. (2008). Pressão alta e stress: O que fazer agora? Um guia de vida para o hipertenso. Campinas: Papirus.

Malagris, L. E. N. (2004). A via L-arginina-óxido nítrico e o controle do stress em pacientes com hipertensão arterial sistêmica. (Unpublished thesis dissertation). Universidade do Estado do Rio de Janeiro, Rio de Janeiro.
Malagris, L. E. N., Brunini, T. M. C., Moss, M. B.,Silva, P. J. A., Espósito, B. R., \& Ribeiro, A. C. M. (2009). Evidências biológicas do treino de controle do stress em pacientes com hipertensão. Psicologia: Reflexão e Crítica, 22(1), 1-9.

Nealey-Moore, J. B., Smith, T. W., Uchino, B. N., Hawkins, M. W., \& Olson-Cerny, C. (2007). Cardiovascular reactivity during positive and negative marital interactions. Journal of Behavioral Medicine, 30(6), 505-519.

Ohira, T. (2010). Psychological distress and cardiovascular disease: the Circulatory Risk in Communities Study (CIRCS). Journal of Epidemiology, 20(3), 185-191.

Parrot, D. J., Zeichner, A., \& Evces, M. (2005). Effect of trait anger on cognitive processing of emotional stimuli. Journal of General Psychology, 132(1), 67-80.

Player, M. S., King, D. E., Mainous, A. G., \& Geesey, M. E. (2007). Psychosocial factors and progression from prehypertension to hypertension or coronary heart disease. Annals of Family Medicine, 5(5), 403-411.

Pointer, M. A., Yancey, S., Abou-Chacra, R., Petrusi, P., Waters, S.J., \& McClelland, M. K. (2012). State Anxiety Is Associated with Cardiovascular Reactivity in Young, Healthy African Americans. International Journal of Hypertension, 1-7. doi:10.1155/2012/268013.

Poole, J. C., Snieder, H., Davis, H. C., \& Treiber, F. A. (2006). Anger suppression and adiposity modulate association between ADRB2 haplotype and cardiovascular stress reactivity Psychosomatic Medicine, 68 (2), 207-212.

Richter, S., Deter, H. C., Rudat, M., Schächinger, H., ZimmermannViehoff, F., \& Weber C. (2011). Anger and cardiovascular startle reactivity in normotensive young males. International Journal of Psychophysiology, 79(3), 364-370.

Sanz, J., García-Vera, M.P., Espinosa, R., Fortún, M., Magán, I., \& Segura, J. (2010). Psychological factors associated with poor hypertension control: differences in personality and stress between patients with controlled and uncontrolled hypertension. Psychological Reports, 107(3), 923-938.

Sociedade Brasileira de Cardiologia (SBC), Sociedade Brasileira de Hipertensão(SBH), Sociedade Brasileira de Nefrologia (SBN) (2010). VI Diretrizes Brasileiras de Hipertensão Arterial. Revista Brasileira de Hipertensão, 17(1), 4-64.

Spielberger, C. D. (1992). Inventário de Expressão de Raiva como Estado e Traço (STAXI) (A. Biaggio, trans.). São Paulo: Vetor Editora Psicopedagógica.

Straub, R. O. (2005). Psicologia da Saúde (R. C. Costa, trans.). Porto Alegre: Artmed Editora.

Treiber, F. A., Kamarck, T., Scheneiderman, N., Sheffield, D., Kapppuku, G., \& Taylor, T. (2003). Cardiovascular reactivity and development of preclinical and clinical disease states. Psychosomatic Medicine, 65(1), 46-62. 